\title{
THE ASYMPTOTIC BEHAVIOR OF A CLASS OF NONLINEAR DIFFERENTIAL EQUATIONS OF SECOND ORDER
}

\author{
JINGCHENG TONG
}

\begin{abstract}
Let $u^{\prime \prime}+f(t, u)=0$ be a nonlinear differential equation. If there are two nonnegative continuous functions $v(t), \varphi(t)$ for $t>0$, and a continuous function $g(u)$ for $u>0$, such that (i) $\int_{1}^{\infty} v(t) \varphi(t) d t<\infty$; (ii) for $u>0, g(u)$ is positive and nondecreasing; (iii) $|f(t, u)|<v(t) \varphi(t) g(|u| / t)$ for $t>1,-\infty<u<$ $\infty$, then the equation has solutions asymptotic to $a+b t$, where $a, b$ are constants and $b \neq 0$. Our result generalizes a theorem of D. S. Cohen [3].
\end{abstract}

Consider the nonlinear differential equation

(1)

$$
u^{\prime \prime}+f(t, u)=0 \text {. }
$$

D. S. Cohen [3] proved the following theorem.

TheOREM A. Suppose $f(t, u)$ satisfies the following conditions:

(H-1) $f(t, u)$ is continuous on $D: t \geqslant 0,-\infty<u<\infty$.

(H-2) the derivative $f_{u}(t, u)$ exists on $D$ and $f_{u}(t, u)>0$ on $D$.

(H-3) $|f(t, u)|<f_{u}(t, 0)|u|$ on $D$.

In addition, suppose that

$$
\int_{1}^{\infty} t f_{u}(t, 0) d t<\infty
$$

Then equation (1) has solutions which are asymptotic to $a+b t$ as $t \rightarrow \infty$, where $a, b$ are constants and $b \neq 0$.

In the proof of Theorem A, Cohen used R. Bellman's method [1, pp. 114-115] and Gronwall's inequality. In this paper we use the same method and Bihari's inequality [2] to generalize Theorem A.

TheOREM B. Let $f(t, u)$ be continuous on $D: t \geqslant 0,-\infty<u<\infty$. If there are two nonnegative continuous functions $v(t), \varphi(t)$ for $t \geqslant 0$, and a continuous function $g(u)$ for $u \geqslant 0$, such that

(i) $\int_{1}^{\infty} v(t) \varphi(t) d t<\infty$,

(ii) for $u>0, g(u)$ is positive and nondecreasing,

(iii) $|f(t, u)|<v(t) \varphi(t) g(|u| / t)$ for $t \geqslant 1,-\infty<u<\infty$,

then the equation (1) has solutions which are asymptotic to $a+b t$, where $a, b$ are constants and $b \neq 0$.

Received by the editors August 11, 1980 and, in revised form, April 15, 1981.

AMS (MOS) subject classifications (1970). Primary 34C10.

Key words and phrases. Gronwall's inequality, Bihari's inequality.

(C) 1982 American Mathematical Society 0002-9939/82/0000-0416/\$01.50 
Remark. If we let $v(t)=f_{u}(t, 0), \varphi(t)=t, g(u)=u$ in Theorem B, we obtain Theorem A.

Proof of TheOrem B. Integrating (1) twice on $[1, t]$, we have

$$
u(t)=c_{1}+c_{2} t-\int_{1}^{t}(t-s) f(s, u(s)) d s .
$$

Choose $c_{1}>1$ and let $c_{3}=c_{1}+\left|c_{2}\right|$. Then for $t>1$ we have

$$
\begin{aligned}
\frac{|u(t)|}{t} & \leqslant c_{3}+\int_{1}^{t} f(s, u(s)) d s \\
& \leqslant c_{3}+\int_{1}^{t} v(s) \varphi(s) g(|u(s)| / s) d s .
\end{aligned}
$$

By Bihari's inequality we have

$$
\frac{|u(t)|}{t} \leqslant G^{-1}\left(G\left(c_{3}\right)+\int_{1}^{t} v(s) \varphi(s) d s\right) .
$$

Here $G(x)=\int_{1}^{x} d t / g(t), G^{-1}(x)$ is the inverse function of $G(x)$. From $g(t)>0$ we know that $G(x)$ is increasing; hence $G^{-1}(x)$ exists, and is also increasing.

Now let $c_{4}=G\left(c_{3}\right)+\int_{1}^{\infty} v(s) \varphi(s) d s$. Since $G^{-1}(x)$ is increasing, we have

Differentiating (3), we have

$$
\frac{|u(t)|}{t} \leqslant G^{-1}\left(c_{4}\right)
$$

$$
u^{\prime}(t)=c_{2}-\int_{1}^{t} f(s, u(s)) d s
$$

By conditions (i), (ii), (iii) and (5), we have

$$
\begin{aligned}
\int_{1}^{t}|f(s, u(s))| d s & \leqslant \int_{1}^{t} v(t) \varphi(t) g(|u(s)| / s) d s \\
& \leqslant g\left(G^{-1}\left(c_{4}\right)\right) \int v(s) \varphi(s) d s<\infty .
\end{aligned}
$$

Therefore $u^{\prime}(t) \rightarrow c_{2}-\int_{1}^{\infty} f(s, u(s)) d s$ as $t \rightarrow \infty$. If we choose $c_{2}$ sufficiently large, then $u^{\prime}(t)>1$. Hence $\lim _{t \rightarrow \infty} u^{\prime}(t) \neq 0$. This proves the theorem.

We give an example to which Theorem B applies but Theorem A does not.

EXAMPLE.

$$
u^{\prime \prime}+t^{-4} u^{2} \cos u=0 .
$$

Since $f_{u}(t, 0)=0$, (H-3) does not hold and Theorem A does not apply. Let $v(t)=t^{-4}, \varphi(t)=t^{2}, g(u)=u^{2}$. Then conditions (i), (ii) and (iii) are satisfied and equation (7) has solutions asymptotic to $a+b t$ as $t \rightarrow \infty$.

ACKNOWLeDgement. The author is indebted to the referee for simplifying the conditions of Theorem B.

\section{REFERENCES}

1. R. Bellman, Stability theory of differential equations, McGraw-Hill, New York, 1953.

2. I. Bihari, A generalization of a lemma of Bellman and its application to uniqueness problems of differential equations, Acta Math. Acad. Sci. Hungar. 7 (1957), 81-94.

3. D. S. Cohen, The asymptotic behavior of a class of nonlinear differential equations, Proc. Amer. Math. Soc. 18 (1967), 607-609.

Department of Mathematics, Wayne State University, Detrort, Michigan 48202

Institute of Mathematics, Academia Sinica, Peking, China 